\title{
WASP-7: A BRIGHT TRANSITING-EXOPLANET SYSTEM IN THE SOUTHERN HEMISPHERE
}

Coel Hellier $^{1}$, D. R. Anderson ${ }^{1}$, M. Gillon ${ }^{2}$, T. A. Lister ${ }^{1,3}$, P. F. L. Maxted ${ }^{1}$, D. Queloz ${ }^{2}$, B. Smalley ${ }^{1}$, A. H. M. J. Triaud $^{2}$, R. G. West $^{4}$, D. M. Wilson ${ }^{1}$, K. Alsubai ${ }^{5}$, S. J. Bentley ${ }^{1}$, A. Collier Cameron ${ }^{5}$, L. HebB ${ }^{5}$, K. Horne ${ }^{5}$, J. Irwin ${ }^{6}$,

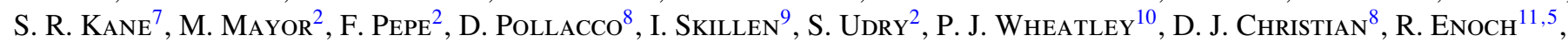
C. A. Haswell ${ }^{11}$, Y. C. Joshi ${ }^{8}$, A. J. Norton ${ }^{11}$, N. Parley ${ }^{11}$, R. Ryans ${ }^{8}$, R. A. Street ${ }^{3,8}$, AND I. Todd ${ }^{8}$

${ }^{1}$ Astrophysics Group, Keele University, Staffordshire, ST5 5BG, UK

${ }^{2}$ Observatoire de Genève, 51 ch. des Maillettes, 1290 Sauverny, Switzerland

${ }^{3}$ Las Cumbres Observatory, 6740 Cortona Dr. Suite 102, Santa Barbara, CA 93117, USA

${ }^{4}$ Department of Physics and Astronomy, University of Leicester, Leicester, LE1 7RH, UK

${ }^{5}$ School of Physics and Astronomy, University of St. Andrews, North Haugh, Fife, KY16 9SS, UK

${ }^{6}$ Department of Astronomy, Harvard University, 60 Garden Street, MS 10, Cambridge, Massachusetts 02138, USA

${ }^{7}$ Michelson Science Center, Caltech, MS 100-22, 770 South Wilson Avenue, Pasadena, CA 91125, USA

${ }^{8}$ Astrophysics Research Centre, School of Mathematics \& Physics, Queen's University, University Road, Belfast, BT7 1NN, UK

${ }^{9}$ Isaac Newton Group of Telescopes, Apartado de Correos 321, E-38700 Santa Cruz de la Palma, Tenerife, Spain ${ }^{10}$ Department of Physics, University of Warwick, Coventry, CV4 7AL, UK

${ }^{11}$ Department of Physics and Astronomy, The Open University, Milton Keynes, MK7 6AA, UK Received 2008 May 17; accepted 2008 November 17; published 2008 December 11

\begin{abstract}
We report that a Jupiter-mass planet, WASP-7b, transits the $V=9.5$ star HD 197286 every $4.95 \mathrm{~d}$. This is the brightest discovery from the WASP-South transit survey so far and is currently the brightest transiting-exoplanet system in the southern hemisphere. WASP-7b is among the densest of the known Jupiter-mass planets, suggesting that it has a massive core. The planet mass is $0.96_{-0.18}^{+0.12} M_{\mathrm{Jup}}$, the radius is $0.915_{-0.040}^{+0.046} R_{\mathrm{Jup}}$, and the density is $1.26_{-0.21}^{+0.25} \rho_{\text {Jup }}\left(1.67_{-0.28}^{+0.33} \mathrm{~g} \mathrm{~cm}^{-3}\right)$.

Key words: stars: individual (WASP-7, HD 197286) - planetary systems
\end{abstract}

\section{INTRODUCTION}

Transiting exoplanets are valuable discoveries since they offer the most opportunities for parameterization and study. The WASP project (Pollacco et al. 2006) is one of the number of wide-area surveys, along with HAT (Bakos et al. 2002), TrES (O'Donovan et al. 2006), and XO (McCullough et al. 2005), all aimed at finding exoplanets transiting relatively bright stars, where they are easiest to observe. A prime aim is to fill out diagrams such as the exoplanet mass-radius plot, which has the potential to be a diagnostic tool for exoplanets comparable to the Hertzsprung-Russell diagram for stars, possibly leading to an understanding of the large disparity in the parameters of known hot Jupiters.

WASP is the only one of the above surveys operating in both hemispheres. We report here on WASP-7 (= HD 197286), a new discovery from WASP-South that, at magnitude 9.5, is currently the brightest transiting-exoplanet system in the southern hemisphere, being three magnitudes brighter than the previously announced WASP-4 (Wilson et al. 2008) and WASP5 (Anderson et al. 2008). WASP-7b is also among the densest known Jupiter-mass exoplanets, extending the populated region of the mass-radius plot.

\section{OBSERVATIONS}

The WASP-South survey is described in Pollacco et al. (2006) and Wilson et al. (2008), while a discussion of our candidate selection methods can be found in Collier Cameron et al. (2007a), Pollacco et al. (2007), and references therein.

WASP-7 ( = HD 197286) is a $V=9.5$ F5V star in Microscopium. It was observed by WASP-South from May to midOctober in both 2006 and 2007, being recorded in two overlapping cameras, each having an $11.1 \mathrm{~cm}$ aperture Canon $200 \mathrm{~mm}$ $\mathrm{f} / 1.8$ lens backed by a $2 \mathrm{k} \times 2 \mathrm{k} e 2 V \mathrm{CCD}$. Exposure times were
$30 \mathrm{~s}$, with a typical cadence of 8 minute. We obtained 5800 photometric data points from each camera in 2006 , and a further 5700 data points from each in 2007.

The WASP-South lightcurves revealed dips with a depth of 0.007 magnitudes recurring with a 4.95 day period (Figure 1). Spectroscopic observations were then obtained using the CORALIE spectrograph on the Euler $1.2 \mathrm{~m}$ telescope. Eleven radial-velocity measurements were obtained during 2007 September 15-October 12 (Figure 2; Table 1), establishing WASP-7b as a Jupiter-mass companion.

We used a line-bisector analysis to look for asymmetries in the spectral line profiles, as could be caused by contamination from an unresolved eclipsing binary (Queloz et al. 2001). Such a binary would produce bisector spans that vary in phase with the photometric period with an amplitude comparable to the radialvelocity amplitude. This is not seen in our data, supporting the conclusion that the radial-velocity variations are caused by a planet.

\section{WASP-7 PARAMETERS}

The CORALIE spectra, when co-added, give a signal-to-noise ratio of $\sim 50$ in $0.01 \AA$ bins, which is suitable for a preliminary photospheric analysis of WASP-7.

We analyzed the spectra using the UCLSYN package and ATLAS9 models, without convective overshooting (Castelli et al. 1997), leading to the parameters in Table 2. The effective temperature $\left(T_{\text {eff }}\right)$ comes from an analysis of the $\mathrm{H} \alpha$ line, while the surface gravity $(\log g)$ comes from the $\mathrm{Na}$ I $\mathrm{D}$ and $\mathrm{Mg}$ I $\mathrm{b}$ lines. An estimate of the microturbulence $\left(\xi_{\mathrm{t}}\right)$ comes from several clean and unblended $\mathrm{Fe} I$ and $\mathrm{Fe}$ II lines, while the ionization balance between $\mathrm{Fe}$ I and Fe II was used as an additional diagnostic of $T_{\text {eff }}$ and $\log g$.

In addition to the spectral analysis, we have also used TYCHO, DENIS, and 2MASS magnitudes to estimate the 

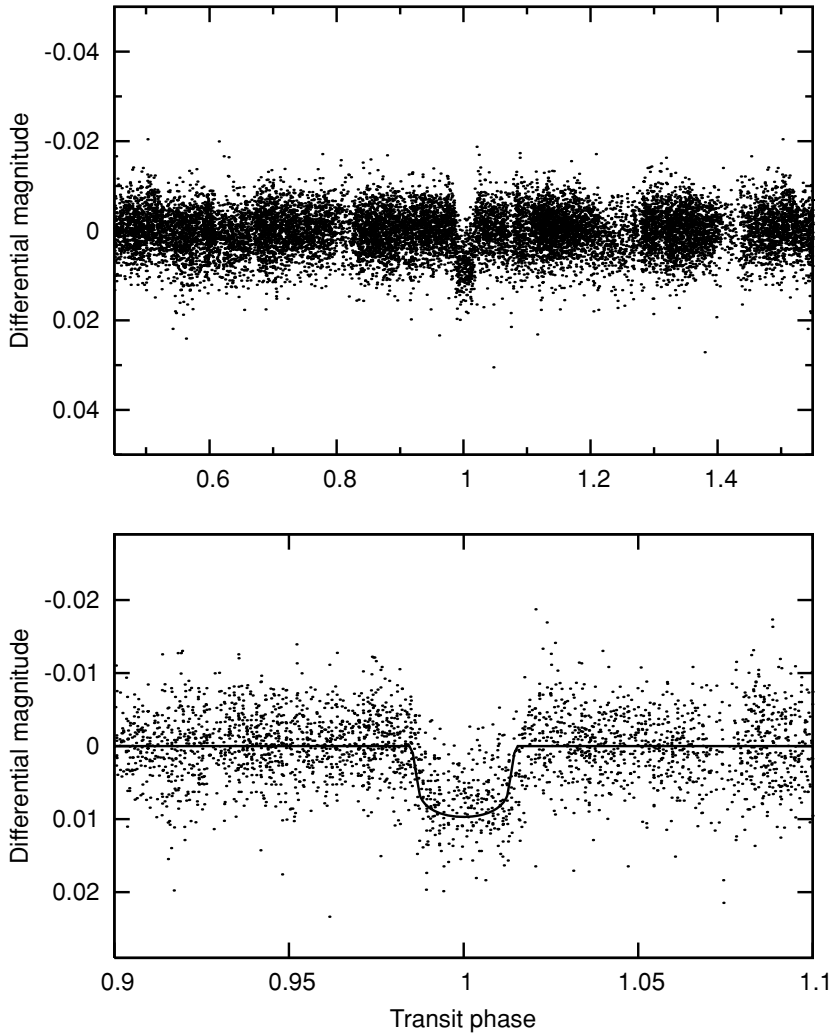

Figure 1. WASP-South lightcurve of WASP-7 folded on the 4.95 day transit period. The lower panel shows the transit phases enlarged, together with the best-fitting model.

effective temperature using the infrared flux method (Blackwell $\&$ Shallis 1977). This gives $T_{\text {eff }}=6370 \pm 150 \mathrm{~K}$, which is in close agreement with that obtained from the spectroscopic analysis. These results are consistent with the F5V spectral-type determined by Houk (1982).

The rotation rate of WASP-7 is $v \sin i=17 \pm 2 \mathrm{~km} \mathrm{~s}^{-1}$. This is in line with expectations for an F5V star (Gray 1992 quotes $\langle v \sin i\rangle=20$ ), but is the largest known among host stars of transiting planets, and leads to the prediction of a large Rossiter-McLaughlin effect of $98 \pm 19 \mathrm{~m} \mathrm{~s}^{-1}$ (e.g., Gaudi \& Winn 2007).

We have investigated whether the star might be chromospherically active; however, there is no sign of $\mathrm{Ca} \mathrm{H}+\mathrm{K}$ line emission in the CORALIE spectra. We have also looked for variability in the WASP data at the predicted rotation period of $3.7 \pm$ 0.5 days, but found no such variability with an upper limit of $0.02 \mathrm{mag}$.

The Li I $6708 \AA$ line is not detected in the CORALIE spectra (EW $\ll 1 \mathrm{~mA}$, allowing us to derive an upper limit on the lithium abundance of $\log \mathrm{n}(\mathrm{Li} / \mathrm{H})+12 \ll 1.0)$. However, the $T_{\text {eff }}$ of this star implies that it is in the lithium gap (Böhm-Vitense 2004), and thus the lithium line does not provide an age constraint.

\subsection{Planetary Parameters}

The CORALIE radial-velocity measurements were combined with the WASP-South photometry in a simultaneous Markovchain Monte Carlo (MCMC) analysis to find the parameters of the WASP-7 system. This process is described in detail in Collier Cameron et al. (2007b) and Pollacco et al. (2007). The optimal MCMC parameters are listed in Table 3.

An initial run found an eccentricity, $e<0.17$, and we then adopted $e=0$ for the solution presented here. In order to
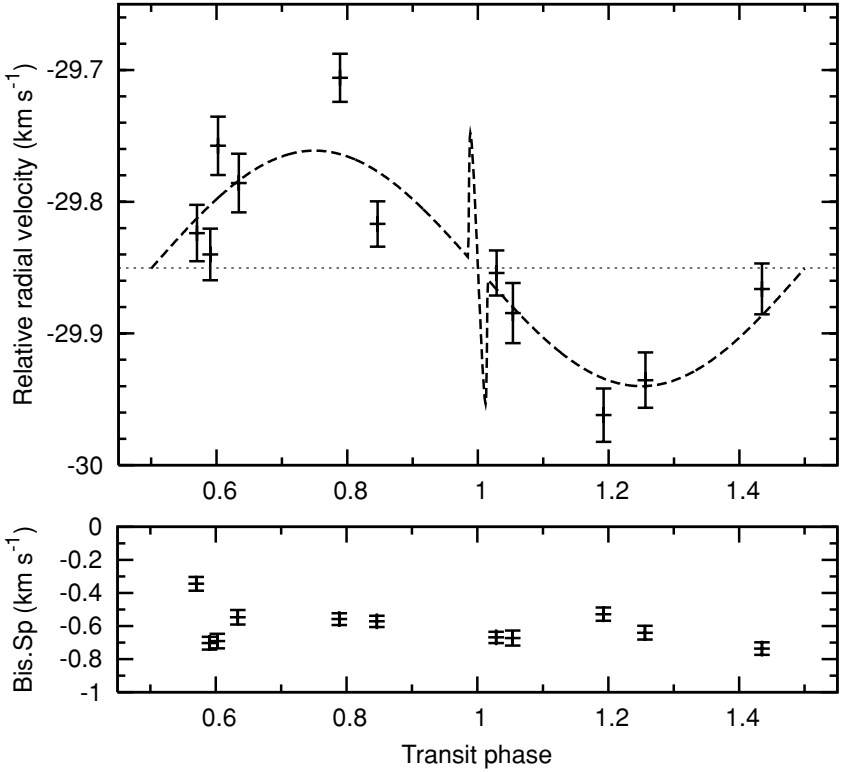

Figure 2. CORALIE radial-velocity curve for WASP-7 along with the bestfitting model (including the predicted Rossiter-McLaughlin effect, which is large for this fast rotator). The lower panel shows the bisector spans.

Table 1

CORALIE Radial Velocities for WASP-7

\begin{tabular}{lccc}
\hline \hline $\begin{array}{l}\text { Time } \\
\text { BJD - 245 0000 }\end{array}$ & $\begin{array}{c}\text { Rad. Vel. } \\
\left(\mathrm{km} \mathrm{s}^{-1}\right)\end{array}$ & $\begin{array}{c}\sigma_{\mathrm{RV}} \\
\left(\mathrm{km} \mathrm{s}^{-1}\right)\end{array}$ & $\begin{array}{c}\text { Bis. Span } \\
\left(\mathrm{km} \mathrm{s}^{-1}\right)^{\mathrm{a}}\end{array}$ \\
\hline 4329.71524 & -29.8238 & 0.0214 & -0.344 \\
4330.79865 & -29.7059 & 0.0182 & -0.558 \\
4359.54361 & -29.8400 & 0.0196 & -0.703 \\
4362.52524 & -29.9620 & 0.0202 & -0.529 \\
4364.55740 & -29.7575 & 0.0221 & -0.691 \\
4376.57881 & -29.8540 & 0.0171 & -0.669 \\
4378.59076 & -29.8662 & 0.0194 & -0.736 \\
4379.57799 & -29.7858 & 0.0222 & -0.547 \\
4380.63008 & -29.8169 & 0.0173 & -0.571 \\
4382.66066 & -29.9355 & 0.0210 & -0.641 \\
4386.61194 & -29.8845 & 0.0229 & -0.673 \\
\hline
\end{tabular}

Note. ${ }^{\text {a }}$ Bisector spans; $\sigma_{\mathrm{BS}} \approx 2 \sigma_{\mathrm{RV}}$

Table 2

Parameters of WASP-7/HD 197286

\begin{tabular}{lc}
\hline \hline & R.A. $=20^{\mathrm{h}} 44^{\mathrm{m}} 10^{\mathrm{s}} 22$, Decl. $=-39^{\circ} 13^{\prime} 30^{\prime \prime} .9$ \\
$T_{\text {eff }}$ & $6400 \pm 100 \mathrm{~K}$ \\
$\log g$ & $4.3 \pm 0.2$ \\
{$[\mathrm{Fe} / \mathrm{H}]$} & $0.0 \pm 0.1$ \\
$\xi_{\mathrm{t}}$ & $1.5 \pm 0.2 \mathrm{~km} \mathrm{~s}^{-1}$ \\
$v \sin i$ & $17 \pm 2 \mathrm{~km} \mathrm{~s}^{-1}$ \\
Spectral type & $\mathrm{F} 5 \mathrm{~V}$ \\
Distance & $140 \pm 15 \mathrm{pc}$ \\
$V$ mag & 9.51 \\
\hline
\end{tabular}

balance the weights of the photometry and radial velocities in the MCMC analysis, we added a systematic error of $20 \mathrm{~m} \mathrm{~s}^{-1}$ in quadrature to the radial velocities (as might arise, for example, from stellar activity) to reconcile $\chi^{2}$ with the number of degrees of freedom. The rms residual to this fit is $0.03 \mathrm{~km} \mathrm{~s}^{-1}$.

WASP-7b is among the densest of the Jupiter-mass planets (Figure 3), which suggests that it has a large core. The calculations presented by Fortney et al. (2007) suggest a core mass of $\approx 100$ Earth masses, or $\approx 0.3$ of the planet, with some dependence on the planetary age. Overall, WASP-7b adds to the finding that hot Jupiters show a wide disparity in densities, from 
Table 3

System Parameters for WASP-7

\begin{tabular}{|c|c|c|}
\hline \multirow{2}{*}{$\begin{array}{l}\text { Parameter } \\
P \text { (days) }\end{array}$} & \multicolumn{2}{|c|}{ Value } \\
\hline & 4.954658 & $\begin{array}{l}+0.000055 \\
-0.000043\end{array}$ \\
\hline$T_{\mathrm{C}}(\mathrm{HJD})$ & 2453985.0149 & $\begin{array}{l}+0.0009 \\
{ }_{-0.0012}\end{array}$ \\
\hline$T_{14}$ (days) $^{\mathrm{a}}$ & 0.1573 & $\begin{array}{l}+0.0024 \\
-0.0018\end{array}$ \\
\hline$R_{\mathrm{P}}^{2} / R_{*}^{2}$ & 0.00579 & $\begin{array}{l}+0.00013 \\
-0.00026\end{array}$ \\
\hline$b \equiv a \cos i / R_{*}\left(R_{\odot}\right)$ & 0.08 & $\begin{array}{l}+0.17 \\
-0.08\end{array}$ \\
\hline$i(\operatorname{degs})$ & 89.6 & ${ }_{-0.9}^{+0.4}$ \\
\hline$e$ & 0 (adopted) & \\
\hline$K_{1}\left(\mathrm{~km} \mathrm{~s}^{-1}\right)$ & 0.097 & $\begin{array}{l}+0.013 \\
{ }_{-0.013}\end{array}$ \\
\hline$\gamma\left(\mathrm{km} \mathrm{s}^{-1}\right)$ & -29.8506 & $\begin{array}{l}+0.0017 \\
{ }_{-0}^{0.0016}\end{array}$ \\
\hline$M_{*}\left(M_{\odot}\right)$ & 1.28 & $\begin{array}{l}+0.09 \\
-0.19\end{array}$ \\
\hline$R_{*}\left(R_{\odot}\right)$ & 1.236 & $\begin{array}{l}+0.059 \\
-0.046\end{array}$ \\
\hline$T_{\text {eff }}(\mathrm{K})$ & 6400 & \pm 100 \\
\hline $\log g_{*}(\mathrm{cgs})$ & 4.363 & ${ }_{-0.047}^{+0.010}$ \\
\hline$M_{\mathrm{P}}\left(M_{\mathrm{J}}\right)$ & 0.96 & $\begin{array}{l}+0.12 \\
-0.18\end{array}$ \\
\hline$R_{\mathrm{P}}\left(R_{\mathrm{J}}\right)$ & 0.915 & $\begin{array}{l}+0.046 \\
-0.040\end{array}$ \\
\hline$\rho_{\mathrm{P}}\left(\rho_{\mathrm{J}}\right)$ & 1.26 & $\begin{array}{l}+0.25 \\
-0.21\end{array}$ \\
\hline$\rho_{\mathrm{P}}\left(\mathrm{g} \mathrm{cm}^{-3}\right)$ & 1.67 & $\begin{array}{l}+0.33 \\
-0.28\end{array}$ \\
\hline$a(\mathrm{AU})$ & 0.0618 & $\begin{array}{l}+0.0014 \\
-0.0033\end{array}$ \\
\hline $\log g_{\mathrm{P}}(\mathrm{cgs})$ & 3.421 & $\begin{array}{l}+0.067 \\
-0.071\end{array}$ \\
\hline$T_{\mathrm{P}}(\mathrm{K})$ & 1379 & $\begin{array}{l}+35 \\
-23\end{array}$ \\
\hline
\end{tabular}

Note. ${ }^{\text {a }} T_{14}$ : duration, time from 1 st to 4 th contact. Errors are $1 \sigma$.

the denser WASP-5b (Anderson et al. 2008) and WASP-7b to the bloated TrES-4 (Mandushev et al. 2007) and WASP-12b (Hebb et al. 2008).

The WASP consortium comprises the Universities of Keele, Leicester, St. Andrews, the Queen's University Belfast, the Open University and the Isaac Newton Group. WASP-South is hosted by the South African Astronomical Observatory and we are grateful for their support and assistance. Funding for WASP comes from consortium universities and from the UK's Science and Technology Facilities Council.

\section{REFERENCES}

Anderson, D. R., et al. 2008, MNRAS, 387, L4

Bakos, G. À., Làzàr, J., Papp, I., Sàri, P., \& Green, E. M. 2002, PASP, 114, 974

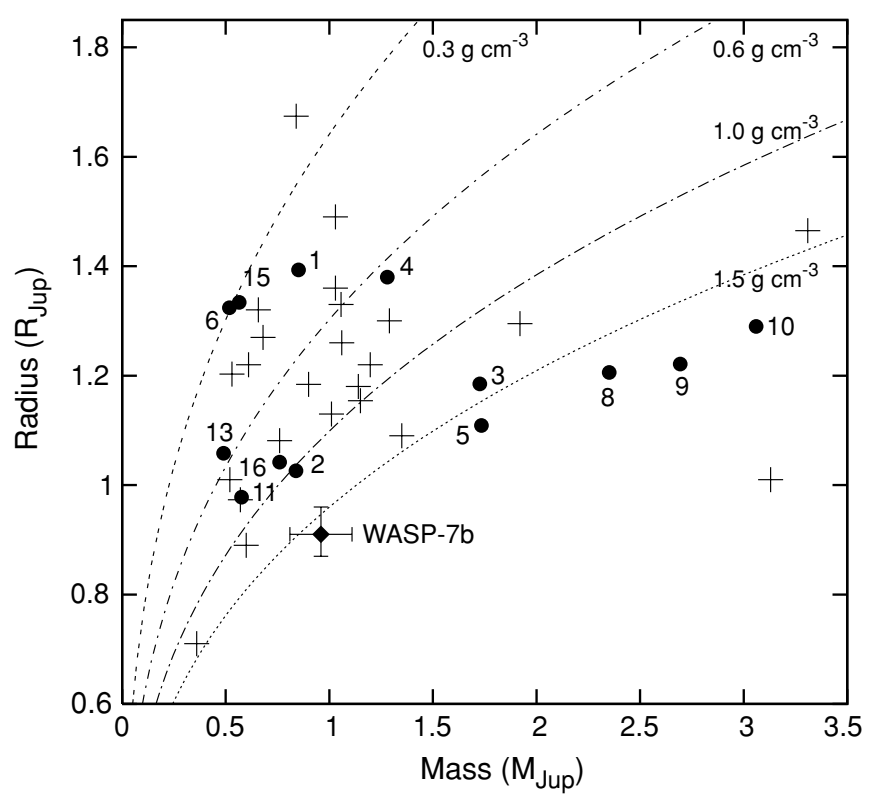

Figure 3. Mass-radius diagram for Jupiter-mass transiting exoplanets. The WASP planets (points) are labelled by their number (data from the WASP consortium, papers in preparation). The remaining data (crosses) are taken from Pont (2008).

Böhm-Vitense, E. 2004, AJ, 128, 2435

Blackwell, D. E., \& Shallis, M. J. 1977, MNRAS, 180, 177

Castelli, F., Gratton, R. G., \& Kurucz, R. L. 1997, A\&A, 318, 841

Collier Cameron, A., et al. 2007a, MNRAS, 375, 951

Collier Cameron, A., et al. 2007b, MNRAS, 380, 1230

Fortney, J. J., Marely, M. S., \& Barnes, J. W. 2007, ApJ, 659, 1661

Gaudi, B. S., \& Winn, J. N. 2007, ApJ, 655, 550

Gray, D. F. 1992, The Observation and Analysis of Stellar Photospheres (2nd ed.; Cambridge: Cambridge Univ. Press)

Hebb, L., et al. 2008, ApJ, submitted

Houk, N. 1982, Catalogue of two-dimensional spectral types for the HD stars, Vol. 3, (Michigan Spectral Survey) (Ann Arbor, MI: Univ. of Michigan) Mandushev, G., et al. 2007, ApJ, 667, L195

McCullough, P. R., Stys, J., Valenti, J., Fleming, S., Janes, K., \& Heasley, J. 2005, PASP, 117, 783

O’Donovan, F. T., et al. 2006, ApJ, 651L, 610

Pollacco, D., et al. 2006, PASP, 118, 1407

Pollacco, D., et al. 2008, MNRAS, 385, 1576

Pont, F. 2008, http://www.inscience.ch/transits/

Queloz, D., et al. 2001, A\&A, 379, 279

Wilson, D. M., et al. 2008, ApJ, 675, L113 